\title{
Fault Recognition of On-Load Tap-Changer Based on Improved BP Neural Network
}

\author{
Chunbing Jiang \\ School of Electrical and Electronic Engineering, North China Electric Power University, Beijing, \\ China \\ Email: jcb808@163.com
}

Keywords: Fault recognition, On-load tap-changer, Neural network, Variable learning rate.

\begin{abstract}
On-load tap-changer (OLTC) is an important part in power transformer, which is used to change the transformation ratio by switching contact form one winding tap to another without interrupting the load. It is necessary to make sure the effective running of OLTC. Thus, a fault recognition method for OLTC based on improved BP neural network is proposed in this paper, according to the working characteristicsof OLTC. The feature of this improved neural network is that the learning rate is variable. Experimental results on 2000 test samples from offline AC test device have showed the effectiveness of the proposed fault recognition method.
\end{abstract}

\section{Introduction}

On-load tap-changer (OLTC) is a kind of device which can be operated under the excitation or load condition of the transformer and is used to exchange the connection position of the winding [1]. The OLTC consists of a switch with a transition impedance and a tap selector [2]. The OLTC is a device which is used to change the number of turns of the first or two coils to change the effective number of turns in the transformer load. The basic principle is to change the transformer winding effectively turns by switching a tap from one to another without interrupting the load current

OLTC fault can be divided into mechanical fault and electrical fault [3-6]. Mechanical fault refers to the fault caused by the failure of the mechanical function of the tap switch, while electrical fault refers to the fault of the electrical performance caused by the deterioration of the electrical components or mechanical failure. The electrical fault reflects the fault source under the combined action of force, electricity and magnetism, and is mainly caused by the contact resistance increasing, contact overheating and so on [7]. The mechanical fault is the main fault of the on load tap changer, and the bad contact is often caused by the failure of the operating mechanism and the failure of the switch. Therefore, it is of great significance and good application prospect for the safe operation of the power system to monitor the mechanical performance, and to recognition the possibility fault of the on load tap changer [8].The test data is provided by the offline AC test device developed by Electric Power Research Institute of Jilin Province.

BP neural network is a multilayer feedforward neural network. The topology of 3-layer BP network is showed in Fig. 1, including input layer, output layer and a hidden layer. The neurons are connected to all neurons in the next layer, while neurons of the same layer have no connections. The basic principle 
of BP neural network is to use gradient descent method to adjust the weights and threshold to minimize the mean square error of the actual output value of the network and the desired output value [9-11]. The traditional BP algorithm does not consider the gradient direction of the previous time in the correction of the weights, making the learning process oscillation and slow convergence. Thus, an improved BP learning algorithm is adopted in this paper by introducing a momentum term to solve the defects of traditional learning algorithm.

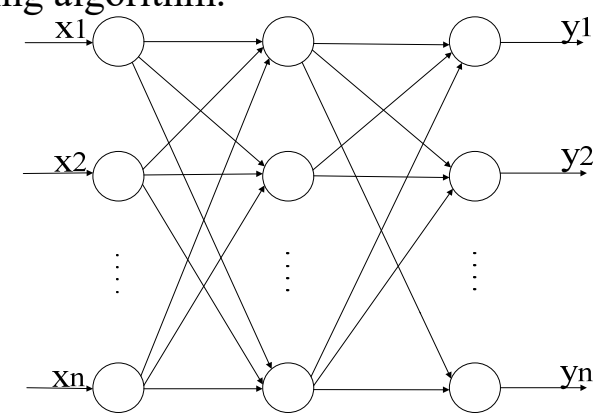

Fig. 1 Topology of 3-layer BP neural network

\section{Improved BP neural network}

The objective function of BP neural network is defined as:

$$
J=\frac{1}{2} \sum_{p=1}^{N} \sum_{k=1}^{m}\left(y_{k}^{p}-o_{k}^{p}\right)^{2}(1)
$$

where $o_{k}^{p}$ is the output of node $k$ under the effect of sample $p$, while $y_{k}^{p}$ is the target value, $m$ is the dimension of output vector, $N$ is the number of training samples.

Training process of BP neural network is to minimize its objective function, and includes the following steps:

(1) Initialization

Set the node number of input layer $n$, node number of hidden layer $l$ and output layer $m$. Set the connection weights between input layer and hidden layer $\omega_{i j}$, and the connection weights between hidden layer and output layer $\omega_{j k}$. Set the thresholds of hidden layer $a=\left[a_{1}, a_{2}, \ldots a_{l}\right]$ and the thresholds of output layer $b=\left[b_{1}, b_{2}, \ldots b_{m}\right]$.

(2) Calculation of hidden layer output $h_{j}$

$$
h_{j}=f\left(\sum_{i=1}^{n} \omega_{i j} x_{i}-a_{j}\right) j=1,2, \ldots, l(2)
$$

(3) Calculation of output layer output $o_{k}$

$$
o_{k}=\sum_{j=1}^{l} h_{j} \omega_{j k}-b_{k}, k=1,2, \ldots, m
$$

(4) Update weights

$$
\begin{gathered}
\omega_{i j}(t+1)=\omega_{i j}(t)+\eta[(1-\beta) D(t)+\beta D(t-1)], t=1,2, \ldots, n(4) \\
\omega_{j k}(t+1)=\omega_{j k}(t)+\eta\left[(1-\beta) D_{1}(t)+\beta D_{1}(t-1)\right], t=1,2, \ldots, n
\end{gathered}
$$

where $\eta$ is the learning rate, and $\eta>0, D(t)=-\partial J / \partial \omega_{i j}(\mathrm{t})$ and $D_{1}(t)=-\partial J / \partial \omega_{j k}(\mathrm{t}), \quad \beta$ is the momentum factor and $0 \leq \beta<1$.

(5) Update thresholds

Update $a_{j}$ and $b_{k}$ by the error of output $o_{k}$ and target output $y_{k}$ : 
(6) Go to step (2) until the mean square error is satisfied.

$$
\begin{array}{r}
a_{j}(t+1)=a_{j}(t)+\eta h_{j}\left(1-h_{j}\right) \sum_{k=1}^{m} \omega_{j k}\left(y_{k}-o_{k}\right)(6) \\
b_{k}(t+1)=b_{k}(t)+\left(y_{k}-o_{k}\right)(7)
\end{array}
$$

In tradition BP neural network, the learning rate is a constant. Value of learning rate has a great effect on the training process. If it is too large, the network will instable. But too small value will lead to slow convergence rate. Thus, we use a new BP neural network in which the learning rate is variable according to the change of mean square error. In training process, if the error decreases, the learning rate increases; otherwise, the learning rate decreases.

$$
\eta(n+1)=\left\{\begin{array}{l}
\operatorname{kinc} \times \eta(n), \text { while } E(n+1)<E(n) \\
k \operatorname{dec} \times \eta(n), \text { while } E(n+1)>E(n)
\end{array}(8)\right.
$$

where kinc and $k d e c$ are increment factor and decrement factor respectively.

\section{Experiments}

\subsection{Dataset}

We use the test data of offline AC test device developed by Electric Power Research Institute of Jilin Province as the experimental dataset. The test data is the current of OLTC in switching process and we select the mean value, variance, mean value of derivative and effective value of the current as the features of test data. We mainly focus on the fault of transition resistance break and poor contact. Thus, we collected 2000 test samples, including 1000 normal samples, 500 resistance break samples and 500 poor contact samples. 1800 test samples are used as training dataset, while the rest samples are the testing dataset.

\subsection{Fault recognition by improved BP neural network}

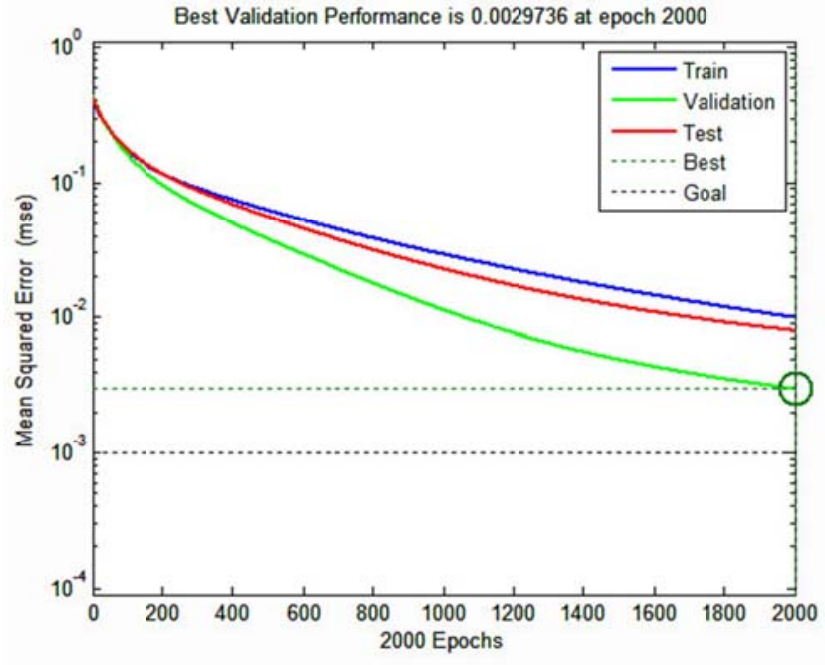

(a) Steepest descent BP neural network

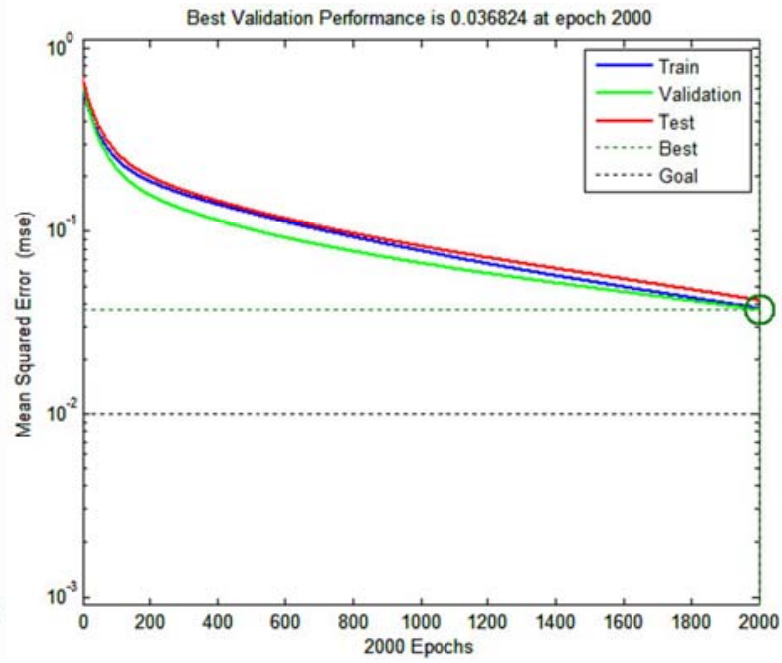

(b) momentum BP neural network

Fig. 2 Experimental result of steepest descent BP neural network and momentum BP neural network 


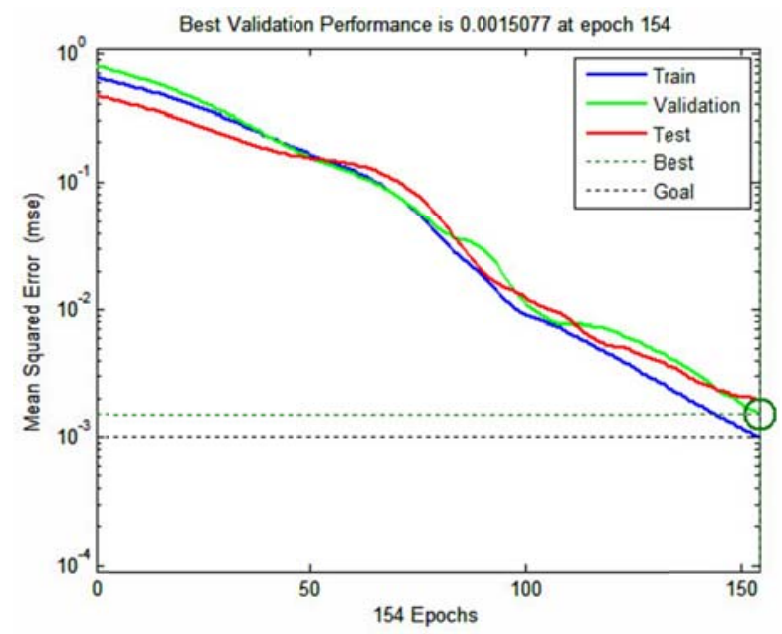

Fig. 3 Experimental result of momentum BP neural network with variable learning rate

Table 1 Testing results of 200 samples

\begin{tabular}{|c|c|c|c|c|}
\hline \multicolumn{2}{|c|}{ Samples } & \multicolumn{3}{c|}{ Output layer } \\
\cline { 3 - 5 } Resistance break & Target value & 1 & 0 & 0 \\
\cline { 2 - 5 } & Actual value & 0.997952 & -0.00382 & 0.010925 \\
\hline \multirow{2}{*}{ Poor contact } & Target value & 0 & 1 & 0 \\
\cline { 2 - 5 } & Actual value & -0.01113 & 1.014515 & -0.01598 \\
\hline \multirow{2}{*}{ Normal } & Target value & 0 & 0 & 1 \\
\cline { 2 - 5 } & Actual value & -0.06778 & -0.01607 & 1.072735 \\
\hline
\end{tabular}

Set the network precision as 0.001 and learning rate as 0.01. Experimental results of steepest descent BP network and momentum BP neural network are showed in Fig. 2. We can find that the two neural networks fail to achieve the setting precision within 2000 steps. Furthermore, in Fig. 2 (a) the testing precision has a great difference with the training precision, illustrating that this kind of network is not suitable for fault recognition of OLTC.

Fig. 3 shows the experimental result of momentum BP neural network with variable learning rate and we can find that this network achieves setting precision only in 154 steps, far faster than the above two networks. All 200 testing samples are convergent, and the results are listed in Table 1. We can find that the actual output values are really closed to the target values, illustrating the effectiveness of proposed fault recognition method.

\section{Conclusions}

In this paper, a fault recognition method for OLTC based on momentum BP neural network with variable learning rate is proposed. This improved network is designed to solve the defects of traditional version. Experimental results showed that the proposed fault recognition method has the ability to recognize the fault of resistance break and poor contact.

\section{Acknowledgements}

We thank the Electric Power Research Institute of Jilin Province for providing experimental data. 


\section{References}

[1] Yao Zhisong, Yao Lei. Practical Manual of On Load Tap Changer [M]. Beijing: China Electric Power Press, 2002.

[2] Meng Ming, Meng Yuanyuan, Xu Changxiang, etc. Fault Diagnosis Model for Power Transformer Based on Support Vector Machine and Dissolved Gas Analysis [J]. Proceedings of the CSEE, 2003, 23 (7): 82-92.

[3] Li Jianpo, Zhao Jiyin, Zheng Ruirui. Fault Diagnosis System on Transformer Based on Gas Chromatography[C]. Proceedings of the 5th International Conference on Machine Learning and Cybernetics. Dalian, China, 2006:809-813.

[4] Yuan Shengfa, Chu Fulei. Support Vector Machines-Based Fault Diagnosis for Turbo-Pump Rotor [J]. Mechanical Systems and Signal Processing, 2006, 20(4):939-952.

[5] Wu Wen, Wang Dazhong, Zhou Zecun, Chen Heng. Application of Artificial Neural Network Combined by Genetic Algorithm in Fault Diagnosis of Power Transformer [J]. Proceedings of the CSEE, 1997, 17 (2): 109-112.

[6] Wang Xiaoxia, Wang Tao. Transformer Fault Diagnosis Based on BP networks [J]. East ChinaNet Electric Power, 2008,36(2):112-116.

[7] Lv Ganyun, Cheng Haozhong, Dong Lixin. Fault Diagnosis of Power Transformer Based on Multi-Layer SVM Classifier [J]. Proceedings of the CSU -EPSA, 2005,17(1):19-22.

[8] Dong Qiguo. Fault Diagnosis of Electric Power Transformer [M]. Beijing: China Electric Power Press, 2001, $10-22$.

[9] Chen Xiaoyu. Application of Improved Neural Network in Transformer Fault Diagnosis [J]. Computer Simulation, 2012,29(8):318-321.

[10] Huang Deshuang. Neural Network Pattern Recognition System Theory [M]. Beijing: Electronic Industry Press, 1996, 105-197.

[11] Yuan Zengren. Artificial Neural Network and Application [M]. Beijing: Tsinghua University Press, 1993, 9-13. 